\title{
Biomechanical Analysis of Volleyball Jump Buffer and Pedal Skills of Top-lever Left-side-hitters from University
}

\author{
Xupeng Wan $^{1}$, Jianzhuang $\mathrm{Niu}^{2}$ \\ 1.P.E Department of Xi'an University of Technology, Xi'an, 710048, China; 2. P.E Department of Xidian
}

University, Xi'an, 710071, China

\begin{abstract}
Jump techniques of five left-side-hitters (who had participated in the China College Volleyball League) are observed and tested by Telemetric EMG testing instrument, Common Speed DV and Synchronizing signal generator. Based on the extensive and interdisciplinary analysis on sports anatomy, exercise science and biomechanics, the relevant parameters with regard to volleyball jump buffer and pedal skills are obtained to help improve high level university volleyball team training.

Keywords: University, Volleyball, Left-side-hitter, Buffer, Pedal, Sports Biomechanics

\section{Participants}

Five professional volleyball players from Northwestern Polytechnical University (one granted the first rank athlete of the nation and others the second) are involved in the research test. All of them have participated in China University Volleyball Association Leagues for several times and received professional training for over five years. The average age is $21.4 \pm 2.19$ years, average height $1.93 \pm 0.06 \mathrm{~m}$, and average weight 83.6 $\pm 6.11 \mathrm{~kg}$. Twenty-four hours before being tested, they are in good physical condition without strenuous exercise or muscle fatigue.

Table 1: The basic information of the participants

\begin{tabular}{ccccc}
\hline & Age & height $(\mathrm{m})$ & Weight $(\mathrm{kg})$ & The level of athletes \\
\hline $\mathrm{Mi}^{* *}$ & 22 & 1.92 & 90 & Grade-2 \\
$\mathrm{Du} * *$ & 20 & 1.88 & 80 & Grade-2 \\
Wang ** & 25 & 2.02 & 85 & Grade-1 \\
$\mathrm{Sun} * *$ & 20 & 1.95 & 88 & Grade-2 \\
$\mathrm{Hu} * *$ & 20 & 1.87 & 75 & Grade-2 \\
\hline
\end{tabular}
\end{abstract}

\section{Methods}

\subsection{Test}

\subsubsection{Instruments and Equipment}

(1)Two Nissan Sony DCR-HC90DV camera, sampling to 50 field/S analysis; (2) a Finland MEGWIN6000 16 channel telemetry EMG, frequency of 1000HZ; (3) a synchronization signal generator; (4) right angle ruler, elastic bandages, razor, $75 \%$ alcohol solution.

\subsection{Document-data method}

The inference to the essays and papers in the library and CNKI is one dispensable way to collect relevant knowledge on volleyball jump buffer and pedal skills of left-side-hitters.

\subsection{Finding Exchanges and Discussions}

Discussions on the testing instruments and data analysis could be conducted with professors and experts from Xi'an Physical Education University and Xi' an University of Electronics and Technology. And an inquiry on jump buffer and pedal skills of left-side-hitters from the professional coaches is another way to help complete the research. 


\section{Results and Analysis}

\subsection{The kinematic analysis of hitting techniques of left-side-hitter}

According to the study, the player's lowest center of gravity divides the whole jumping stage into buffer stage in which muscles complete eccentric contraction and take-off stage in which muscles complete centripetal contraction.

\subsubsection{Comparison of players' time spending on buffer and pedal}

Table 2: The buffer and pedal time of testees' lower limbs in jumping stage

\begin{tabular}{ccccccc}
\hline & Buffer(right) & buffer(left) & pedal(right) & pedal (left) & buffer(all) & pedal(all) \\
\hline Mi ** & 0.16 & 0.00 & 0.14 & 0.12 & 0.16 & 0.14 \\
Du** & 0.12 & 0.00 & 0.20 & 0.14 & 0.12 & 0.20 \\
Wang ** & 0.22 & 0.00 & 0.20 & 0.20 & 0.22 & 0.20 \\
Sun ** & 0.16 & 0.00 & 0.20 & 0.14 & 0.16 & 0.20 \\
Hu ** & 0.16 & 0.00 & 0.14 & 0.12 & 0.16 & 0.14 \\
\hline
\end{tabular}

Unit (Second), similarly hereinafter

We can get the results from table 2 that only Wang's left limb is involved in jump buffer for only 0.02 seconds and his left limb is involved in the whole pedal stage. Whereas, other four testees' left limbs are not involved in buffer stage and their right limbs are partly involved in the pedal procedure. All testees adopt vertical jump with two feet forward. Overall, the testees' time taken to pedal is longer than time taken to pedal. Qinghua Zhang thinks time taken to pedal and the height of the jump is a moderate negative correlation $(\mathrm{R}=-0.58, \mathrm{P}<0.05)$, which means that the shorter time to pedal, the better [1]. However, as far as I'm concerned, the reduction of time to pedal could influence the arm swing, so the height of jumping could be limited.

\subsubsection{The angle parameters of lowest center of gravity point}

Table 3: Comparison of testees' angle parameters of lowest center of gravity

\begin{tabular}{ccccc}
\hline & Angle of left knee & Angle of right knee & Angle of left innominatum & Angle of right innominatum \\
\hline Mi ** & 156.7 & 133.1 & 144.5 & 144.3 \\
$\mathrm{Du} * *$ & 130.8 & 134.3 & 129.3 & 124.1 \\
Wang ** & 163.2 & 119.1 & 152.7 & 135.6 \\
Sun ** & 133.9 & 151.5 & 137.3 & 148.1 \\
$\mathrm{Hu} * *$ & 161.1 & 136.8 & 150.0 & 154.1 \\
Average & 149.1 & 134.9 & 142.7 & 141.2 \\
\hline
\end{tabular}

Unit degree, similarly hereinafter

In general, the figures in table 3 indicate that the bending degree of testees' right knees is greater than their left knees. Ling He believes that the knee bending at 135 degrees is most conducive to enable the extensor muscle groups around knees to generate power [2]. The research data shows that the testees' right knee buffer is better; the angle of Du is 129.3 degree and that of Mi is 124.1 degree. The bigger the hip joint folds [3], the smaller loss of horizontal velocity, which can give full play to dash jump spike effect. 


\subsubsection{The speed value of right foot stepping on ground and feet leaving the ground in take-off stage}

Table 4: the speed value of right foot stepping on ground and feet leaving ground in take-off stage

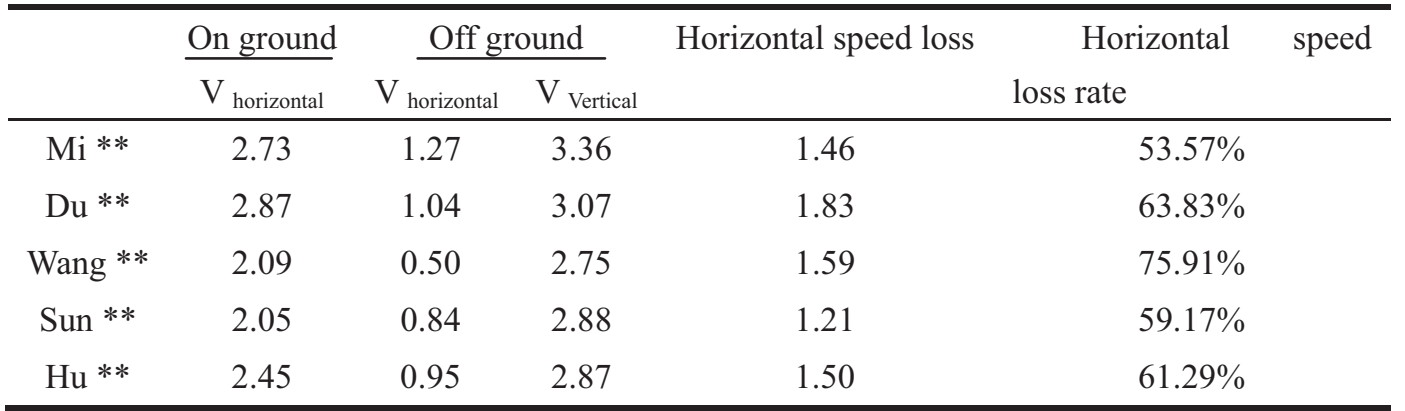

$\mathrm{V}_{\text {horizontal }}$ means horizontal speed; $\mathrm{V}_{\text {Vertical }}$ means Vertical speed; Horizontal speed loss $=$ horizontal speed of right foot stepping on ground -horizontal speed of feet leaving ground; Horizontal speed loss rate= horizontal speed loss/ horizontal speed of right foot stepping on ground [4]

The statistics in Table 4 indicates that Mi's horizontal speed loss rate reaches $53.57 \%$, which is smaller than that of other four athletes. It shows that Mi makes full use of the horizontal speed in take-off stage, which ensures the jumping height under the premise to reduce loss of horizontal speed and can effectively put dash jump spike effect into full play.

\subsection{The analysis of jump muscle mechanics of left-side-hitter}

\subsubsection{EMG activity maps in buffer stage}

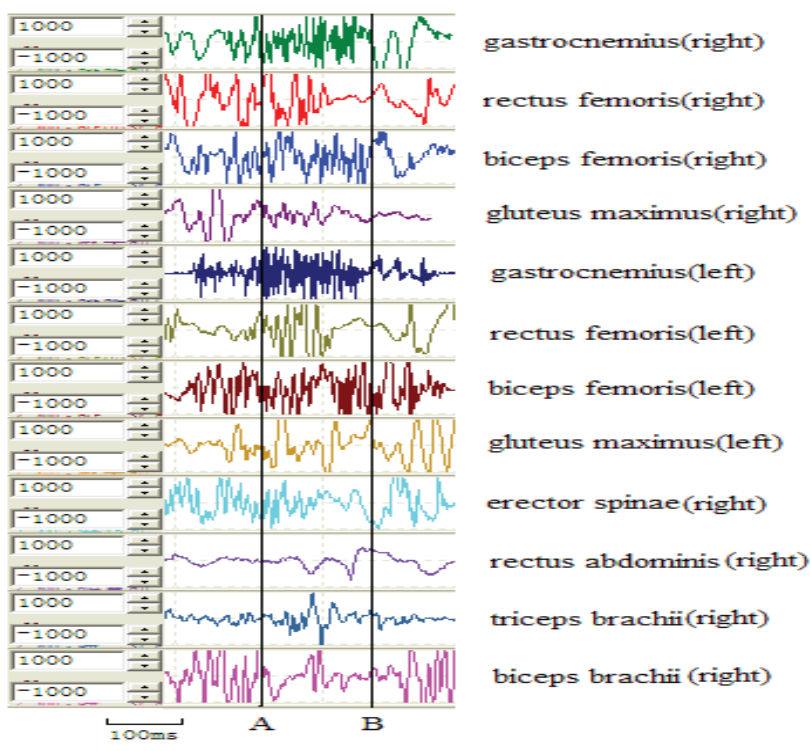

Fig. 1:12 muscle surface EMG of Mi in buffer stage

A: The time when right foot steps on the ground; B: The lowest center of gravity point

It can be seen from Fig. 1 that the measured muscles show higher excitability except rectus muscles in jump buffer stage, especially the right legs' muscles which are consistent with the requirements of motion characteristics: When knees are bent to buffer, on attempt to resist the body to squat, right lower extremity extensor group get into the passive extension work state. EMG value of most muscles tend to reach zero line in "Lowest center of gravity point", and special electronic discharge feature means that muscles are in isometric work state [5]. 


\subsubsection{EMG activity maps in pedal phase}

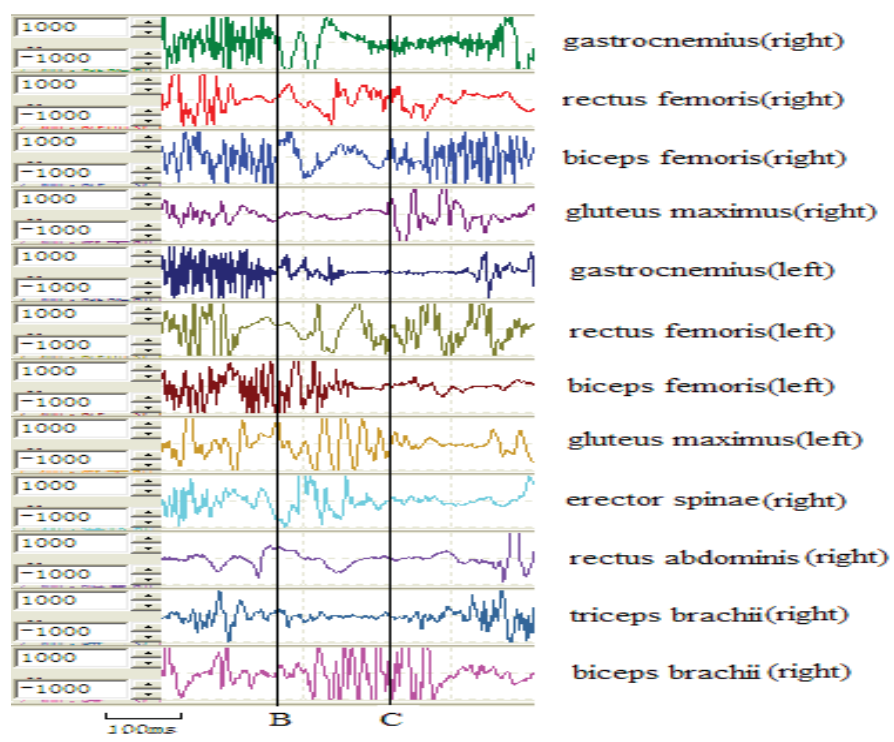

Fig. 2:12 muscle surface EMG of Mi in pedal stage

B: The lowest center of gravity point; C: The time when the feet take off In order to clearly observe the differences and regular patterns of higher excitability of the measured muscles [6] (Fig. 2), the map is transferred to volume chart for better illustration.

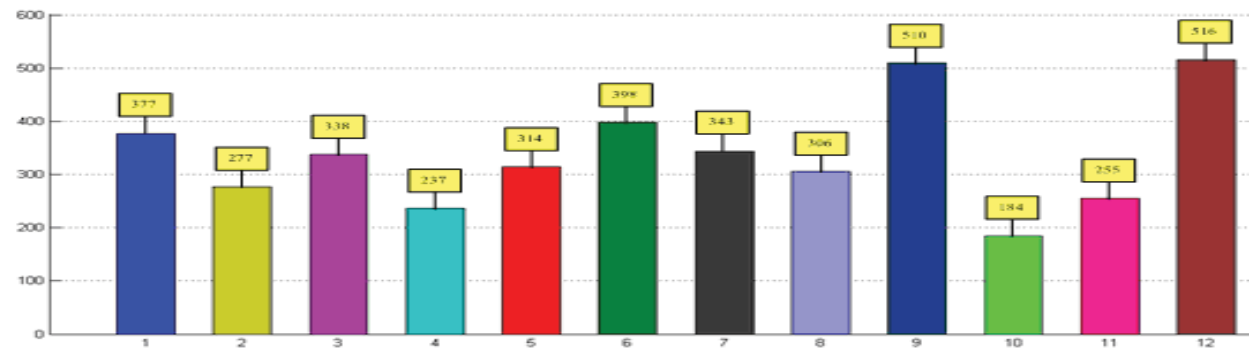

Fig. 3: each muscle integrated EMG value of testees in jump buffer stage

We can find from Fig.3 that the integrated EMG value of right biceps brachii is maximum (516uvs) and that of the right abdominal rectus muscle is minimum (184uvs). Ling He thinks: the jump technique is completed with the cooperation of the arm swing and the legs straightened in the hip joint and knee joint. Therefore, the biceps brachii, which works together to accomplish the jump procedures: arm swing with bent arm by keeping elbows closer to the body, is the main muscle that generates power because its excitability value and discharge value are maximum.

\section{Conclusion}

\subsection{Kinematics}

At the lowest center of gravity point, when the athletes' knee joint angle is around 135 degrees it is most conducive for muscle group to generate power. A proper increase of hip folding range and reduction of the horizontal speed loss could help make a strong attack.

\subsection{Muscle mechanics}

At the lowest center of gravity point, the measured muscles are performing isometric contractions, the direct observation of which lies in that EMG value of most muscles tend to reach zero line. In pedal phase, with lower limb stretches, the biceps brachii, which works together to accomplish the jump procedures: arm swing with bent arm by keeping elbows closer to the body, turns out to be most excitable and become the main muscle that generates power.

\subsection{The analysis of the degree of excitability of biceps brachii}

The study on testing the excitability of right biceps brachii objectively reveals the significance of the wide arm swing technique among the jump techniques for left-side-hitters. The essay aims to remind college volleyball coaches and 
athletes that in the jump stage, especially in the take-off stage, the wide arm swing would be beneficial to help complete the take-off action.

\section{Author Introduction}

Author Introduction: 1.Xupeng Wan, Male, Born in June 1983 in Linyi, Shandong, Master, Lecture, Research Direction: Sports Biomechanics and Theories of Sport Training;2.Jianzhuang Niu, Male, Born in April 1960 in Gong County, Henan, Master, Professor, Research Direction: Sports Biomechanic

\section{References}

[1] Qinghua Zhang, Man volleyball spike jump technique of biomechanical analysis. Sports Sciences Researches, 12 (2), pp. 34-36, 2008.

[2] Ling He, The volleyball back-line spike biomechanical analysis of the main technical links. Journal of Chengdu Sport University, 25 (4), pp. 33-36, 1999.

[3] Shiming Li, Beach volleyball spike jumping characteristics of phase. Journal of Beijing Sport University, 27 (9), pp. 28-29, 2004.

[4] Yijun Li, Chinese and foreign elite man's volleyball players Front Spiking Technique of 3D kinematics. Journal of Xi'an Physical Education University, 17 (3), pp. 36-39, 2000.

[5] Jiaying Zhou, Jump outstretched to anterior lower leg measured muscle surface EMG activity characteristics. Journal of Xi'an Physical Education University, 25 (6), pp. 68-72, 2008.

[6] Shengyu Hu, Sports anatomy, People's education press: Beijing, pp. 107-142, 2000. 